\title{
Bilateral symmetrical cortical osteolytic lesions in two patients with Gaucher disease
}

\author{
Ian M Oppenheim • Astrid Medina Canon • \\ William Barcenas • Catherine Groden • \\ Ozlem Goker-Alpan • Charles S Resnik • \\ Ellen Sidransky
}

Received: 13 April 2011 /Revised: 5 August 2011 / Accepted: 15 August 2011 /Published online: 20 September 2011

(C) Springer-Verlag (outside the USA) 2011

\begin{abstract}
Gaucher disease (GD) is an autosomal recessive lysosomal storage disorder characterized by the reduced or absent activity of glucocerebrosidase. The disease is split into three types. Type 3, or chronic neuronopathic GD, manifests with heterogeneous clinical presentations. Skeletal manifestations of GD can include abnormal bone remodeling resulting in the characteristic Erlenmeyer flask deformities, painful bone crises, osteopenia, and an increased frequency of fractures. Osteolytic lesions can also occur but are rare and tend to be large, expanding intramedullary lesions with cortical thinning. We present two adolescent patients with type 3 GD who developed bilateral symmetrical cortical osteolytic lesions. The lesions in both cases demonstrate predominant cortical scalloping with fairly indolent growth. Neither patient manifests some of the more common bony manifestations of GD-bone crises or osteonecrosis. These atypical and unique skeletal
\end{abstract}

I. M. Oppenheim · C. Groden • O. Goker-Alpan •

E. Sidransky $(\bowtie)$

Medical Genetics Branch, National Human Genome Research Institute, National Institutes of Health,

Building 35, Room 1A213, 35 Convent Drive, MSC 3708, Bethesda, MD 20892-3708, USA

e-mail: sidranse@mail.nih.gov

\section{A. M. Canon}

Hospital Infantil de San José, Fundación Cardioinfantil,

Bogotá, Colombia

\section{W. Barcenas}

Barranquilla Seguro Social,

Barranquilla, Colombia

\section{S. Resnik}

Department of Diagnostic Radiology, University of Maryland School of Medicine,

Baltimore, MD, USA findings in two unrelated probands with type 3 GD further expand the extent of phenotypic variation encountered in this single gene disorder.

Keywords Type 3 Gaucher disease - Osteolytic - Genotype L444P/L444P · Glucocerebrosidase · Gaucher cells

\section{Introduction}

Gaucher disease (GD) is an autosomal recessive lysosomal storage disorder characterized by the reduced or absent activity of glucocerebrosidase. It is a disorder of the reticuloendothelial system, where the deficient enzyme activity causes lipids to accumulate in macrophages, which develop the classic appearance of the Gaucher cell [1, 2]. It has been traditionally divided into three phenotypic types: non-neuronopathic (type 1), acute neuronopathic (type 2), and chronic neuronopathic (type 3) [3]. Type 1 GD, the most common form in the United States, lacks the neuronopathies of type 3 GD such as absent horizontal saccades, dementia, or myoclonus. Both type 1 and type 3 GD have similar hematologic, visceral, pulmonary, or skeletal symptoms, and there is wide phenotypic variance within each type.

The clinical presentation of type $3 \mathrm{GD}$ is heterogeneous, and the onset of symptoms is usually during childhood. Skeletal manifestations can include abnormal bone remodeling resulting in the characteristic Erlenmeyer flask deformities, painful bone crises, osteopenia, and an increased frequency of fractures. Osteolytic lesions can also occur but are rare and tend to be large, expanding intramedullary lesions with cortical thinning [4-6].

Patients with type 3 GD generally show marked improvement in hematological and visceral symptoms 